\title{
Questes
}

Revue pluridisciplinaire d'études médiévales

\section{Les associations de quartier : repenser la communauté politique lucquoise à la fin du XIV siècle}

Diane Chamboduc de Saint Pulgent

\section{(2) OpenEdition}

\section{Journals}

Édition électronique

URL : http://journals.openedition.org/questes/4335

DOI : 10.4000/questes.4335

ISSN : 2109-9472

\section{Éditeur}

Les Amis de Questes

\section{Édition imprimée}

Date de publication : 6 mai 2016

Pagination : 103-122

ISSN : 2102-7188

\section{Référence électronique}

Diane Chamboduc de Saint Pulgent, «Les associations de quartier : repenser la communauté

politique lucquoise à la fin du XIVe siècle », Questes [En ligne], 32 | 2016, mis en ligne le 10 mai 2016, consulté le 06 mai 2019. URL : http://journals.openedition.org/questes/4335 ; DOI : 10.4000/ questes. 4335 


\title{
Les associations de quartier : repenser la communauté politique lucquoise à la fin du XIV $^{\mathbf{e}}$ siècle
}

\author{
Diane Chamboduc de Saint Pulgent \\ Université Paris-Sorbonne
}

Malgré les travaux fondateurs de la sociologie allemande ${ }^{1}$, et après plus d'un siècle de community studies dans la sociologie anglo-saxonne ${ }^{2}$, le terme communauté continue de se caractériser par son relatif flou conceptuel $^{3}$. Le dictionnaire de l'Académie française donne les sens suivants :

état, caractère de ce qui est commun ;

groupe humain dont les membres sont unis par un lien social ;

par extension, groupe humain dont les membres ont en commun une langue, une religion, etc. ;

en zoologie, ensemble d'êtres, de la même espèce ou d'espèces différentes, qui vivent

\footnotetext{
1 Ferdinand Tönnies, Communauté et Société. Catégories fondamentales de la sociologie [1887], introd. et trad. Joseph Leif, Paris, PUF, 1944 et Max Weber, Économie et Société [1921], trad. Julien Freund, Pierre Kamnitzer, Pierre Bertrand, Éric de Dampierre, Éric Maillard et Jacques Chavy, Paris, Plon, 1971. Dans cette tradition, la communauté (Gemeinschaft) s'oppose à la société (Gesellschaft) et les liens entre les deux se comprennent dans le cadre plus large de l'étude des racines du capitalisme et de la modernité.

${ }^{2}$ Cherry Schrecker, «Le concept de communauté dans la sociologie anglo-saxonne », Diversité, 150, septembre 2007, p. 57-64.

${ }^{3}$ En 1955, Georges Hillery identifiait seize définitions différentes du terme dans la littérature scientifique, dont le seul point commun était que la communauté y impliquait toujours des personnes. Voir Georges A. Hillery, «Definitions of Community. Areas of Agreement », Rural Sociology, vol. 20, $\mathrm{n}^{\circ}$ 1, 1955, p. 111-123.
} 
groupés dans un espace donné à une époque déterminée ${ }^{4}$.

En histoire médiévale comme ailleurs, les travaux sur les communautés se sont souvent intéressés aux communautés rurales, parce que les villes, surtout grandes, étaient perçues comme un cadre moins susceptible de susciter chez leurs habitants le sentiment d'appartenance et les traditions partagées au cœur de la définition de la communauté. De la même façon, a souvent été privilégié le moment de formation de ces dernières $^{5}$. L'étude de la communauté politique lucquoise qui suit, à partir des associations de quartiers de la ville, a pour cadre temporel la fin du XIV ${ }^{\mathrm{e}}$ siècle, entre 1369, année de la libération de la ville de la domination pisane, et 1400, date de la prise de pouvoir de Paolo Guinigi, qui devient alors Seigneur de la ville jusqu'en 1430. Elle repose sur trois présupposés différents. Tout d'abord, il ne s'agit pas d'étudier la formation d'une communauté mais son fonctionnement, à un moment certes de reconstruction politique après quarante-deux ans de domination étrangère, mais alors que les institutions politiques lucquoises ont une organisation déjà bien huilée. D'autre part, l'attention portée aux associations de quartier vise à décentrer le regard pour étudier la participation politique des milieux populaires lucquois, difficilement visible dans les institutions centrales de la ville. Il s'agit ainsi de considérer le niveau local comme premier échelon de la vie politique urbaine, dans un contexte d'oligarchisation croissante des différents

\footnotetext{
${ }^{4}$ Le dictionnaire de l'Académie donne aussi des définitions dans le champ religieux. Voir en ligne (consulté le 25 juin 2015) : http://atilf.atilf.fr/dendien/scripts/generic/ cherche.exe? $15 ; \mathrm{s}=3626437365$.

${ }^{5}$ L'ouvrage de Chris Wickham consacré à la formation des communautés rurales dans la campagne lucquoise est un bon exemple de cette double tendance. Voir Chris Wickham, Comunità e clientele nella Toscana del XII secolo. Le origini del comune rurale nella Piana di Lucca, Roma, Viella, 1995 ; traduit en français sous le titre: Communautés et clientèles en Toscane au XII siècle. Les origines de la commune rurale dans la région de Lucques, Rennes, Associations d'Histoire des Sociétés rurales, 2001.
} 
conseils lucquois. Enfin, suivant les recommandations de Vincent Azoulay pour l'étude des cités grecques, il convient, en s'intéressant à ces associations de quartier, de mener de front l'étude du et de la politique. En effet, comme le dit l'introduction du numéro des Annales consacré à la politique en Grèce ancienne,

la politique peut être définie comme un processus de décision publique, fondée sur des formes de délibération collective, se déroulant dans un cadre institutionnel formalisé. [...] À l'inverse, le politique désigne un champ d'action diffus et non institutionnalisé, recouvrant l'ensemble des discours, des rituels et des pratiques collectives qui contribuent à forger, au sein de la communauté, un sentiment d'appartenance partagée - processions, sacrifices, fêtes, banquets, distributions, chœurs ou représentations thêâtrales... ${ }^{6}$

Comme nous le verrons plus bas, les associations de quartier faisaient partie des institutions de la République lucquoise : elles étaient le lieu de délibérations collectives dans un cadre institutionnel formalisé aboutissant à la prise de décisions publiques, même si nous n'avons pas conservé de traces écrites de cette formalisation, sous la forme de statuts par exemple. C'est essentiellement à cette dimension institutionnelle que nous allons nous intéresser dans cet article. Mais les quartiers étaient également l'un des lieux où se formait le sentiment d'appartenance à la communauté urbaine, notamment pour les étrangers installés à Lucques, par le biais de la participation ritualisée aux fêtes et processions de la ville mais aussi de l'expérience de la gestion des propriétés collectives du quartier et, pour les citoyens, de la participation à la milice. Ainsi constituent-ils un thêâtre privilégié pour l'observation et l'étude de la communauté politique lucquoise, notamment parce qu'ils permettent

\footnotetext{
${ }^{6}$ Vincent Azoulay, «Repenser le politique en Grèce ancienne », Annales. Histoire, sciences sociales, vol. 69, n ${ }^{\circ} 3,2014$, p. 605-626, cit. p. 619.
} 
d'apercevoir des acteurs écartés des institutions centrales de la République et donc invisibles dans les sources en émanant.

\section{Le contractualisme politique lucquois}

À la fin du XIV ${ }^{\mathrm{e}}$ siècle, les associations de quartier ne sont pas une nouveauté dans l'organisation urbaine lucquoise. Au XIII siècle, ces groupes, alors clairement dotés d'une dimension militaire et nés, d'après Alma Poloni, de la formalisation et de l'institutionnalisation de liens de solidarité dérivant de la vie en commun dans un même voisinage, de la fréquentation d'une même paroisse et de l'habitude de faire son service militaire dans une même unité opérationnelle de fantassins, avaient constitué la première trame de l'organisation populaire de la ville ${ }^{7}$. Ils n'ont pas laissé de traces archivistiques cohérentes permettant d'en faire l'histoire, mais quelques bribes, dans les archives notariées de la ville ${ }^{8}$.

Les actes étudiés qui concernent des réunions de ces associations frappent aussi bien par leur homogénéité d'ensemble que par leurs variantes dans les détails. Ces comptes rendus débutent par la

\footnotetext{
${ }^{7}$ Alma Poloni, «Strutturazione del mondo corporativo e affermazione del Popolo a Lucca nel Duecento », Archivio Storico Italiano, Firenze, Olschki, 2007, vol. CLXV, $\mathrm{n}^{\mathrm{o}}$ 613, Disp. III, p. 449-486.

${ }^{8}$ Le dépouillement, dans le cadre de ma thèse de doctorat sur les milieux populaires lucquois de la fin du XIV ${ }^{\mathrm{e}}$ siècle, d'environ $20 \%$ des registres de notaires conservés pour l'ensemble de la période à Lucques a ainsi permis d'identifier dix-sept actes inédits enregistrant les réunions de dix associations de quartier de la ville: le «bracchio » San Andrea in Pellaria (deux actes de 1367 et 1375), celui de Santa Lucia verso l'Arco (deux actes de 1393 et 1394), la «contrada» San Jacopo de Tumba (cinq actes de 1373, 1384, 1386, 1387 et 1389), celle de San Alessandro Minore (un acte de 1394), celle de San Masseo (un acte de 1394), celle de San Giovanni Maggiore (un acte de 1394), celle de Santa Lucia verso Fillungho (un acte de 1394), celle de San Martino (un acte de 1394), celle de San Domino (deux actes de 1394 et 1395) et enfin celle de San Giusto (un acte de 1394). Ces actes de nature et de forme variées ont pu être complétés par d'autres, beaucoup plus nombreux, enregistrant plutôt des transactions, en général économiques, comme des contrats de location ou des legs, engageant les quartiers. Ont enfin été utilisées des listes de population, justement dressées par contrada ou par brachio, établies entre 1370 et 1372 sur l'ordre des autorités urbaines.
} 
convocation des habitants du quartier à une réunion, dont le motif est le plus souvent l'élection de représentants (dans quinze cas sur dix-sept). Sont alors précisés, dans neuf cas, le nom de ceux qui ont lancé la convocation, ainsi que le lieu où se tient la réunion, habituellement l'église du quartier (dans huit cas sur les neuf). Puis, dans la totalité des actes étudiés, est dressée la liste des présents, dont il est précisé, dans onze cas, qu'ils représentent plus des deux tiers des habitants (ce qui semble être le quorum nécessaire pour qu'une réunion soit valide) et, dans six cas, qu'ils en constituent la «maior et sanior pars ${ }^{9} »$. Puis, le compte rendu évoque l'élection elle-même, en précisant souvent que les présents ont voté en leur nom et en celui des absents (de manière explicite dans six cas, et implicite dans cinq) et que la décision a été prise avec la concorde de tous et sans opposition (dans quatorze cas). Enfin, les douze actes concernant l'élection d'un consul mentionnent son salaire, dix en précisent le montant, neuf évoquent les habitants chargés de son versement et trois les modalités de ce dernier. Les principales variations d'un acte à l'autre concernent l'évocation des droits et des devoirs des représentants vis-à-vis du quartier et inversement. Alors que les trois actes conservés enregistrant l'élection de procurateurs-syndics sont très détaillés et très similaires les uns aux autres ${ }^{10}$, ceux validant l'élection de consuls sont plus variés et parfois beaucoup plus courts ${ }^{11}$.

\footnotetext{
${ }^{9}$ Comme l'a montré Julien Théry, le vote ne vient qu'en deuxième choix dans les modes de désignation au Moyen Âge, quand l'unanimité n'a pu être atteinte spontanément. Trois modes de scrutin se détachent, comme le concile de Latran IV l'établit clairement : le vote à l'unanimité (qui se distingue de l'unanimité spontanée par la présence d'un débat précédant l'élection, la tractatio), celui à la majorité et enfin celui à la «saniorité », c'est-à-dire suivant l'avis de la «sanior pars », la minorité la plus sage. La plupart des textes médiévaux toutefois, y compris ceux qui s'appuyaient sur Latran IV, estimaient que les deux critères devaient être réunis, comme c'est le cas ici. Voir Julien Théry, « Moyen Âge », dans Dictionnaire du vote, dir. Pascal Perrineau, Dominique Reynié, Paris, PUF, 2001, p. 667-678, p. 670.

${ }^{10}$ Archivio di Stato di Lucca (ASL), Archivio notarii, $\mathrm{n}^{\mathrm{o}} 292, \mathrm{f}^{\mathrm{0}} 116 \mathrm{r}^{\circ}-117 \mathrm{r}^{\mathrm{o}}$, 24 juin 1384.
} 
Le plus frappant reste la forme que ces actes adoptent : il s'agit in fine de contrats liant la communauté du quartier à son ou ses représentants nouvellement élus ${ }^{12}$. Les différents actes visent donc à rappeler les obligations mutuelles des uns et des autres et les contreparties prévues en cas de non-respect de ces dernières : ils se présentent comme des engagements réciproques entre les habitants et leurs représentants. En ce sens-là, ces associations de quartier s'inscrivent dans le continuum contractuel mis en valeur par Paolo Prodi dans son étude sur le serment politique en Occident ${ }^{13}$. Ce recours au contrat dans la gestion des affaires courantes du quartier ou de l'église rappelle donc la diffusion du contractualisme comme fondement de la légitimité politique dans l'Europe de la fin du Moyen Âge et souligne la circulation des formes de gouvernement du sommet de l'État à ses échelons inférieurs et inversement ${ }^{14}$. Ces actes rappellent par ailleurs en partie, dans leur contenu, l'héritage du mouvement populaire qui a triomphé au XIII ${ }^{\mathrm{e}}$ siècle en Italie: les représentants sont avant tout élus pour protéger la communauté et ses biens contre les dommages qui pourraient leur être infligés et pour la représenter devant les cours de justice. Sont implicitement invoqués les principaux mots d'ordre du Popolo et, en

ASL, Archivio notarii, $\mathrm{n}^{\mathrm{o}} 293, \mathrm{f}^{\mathrm{o}} 182 \mathrm{r}^{\circ}-183 \mathrm{v}^{\mathrm{o}}, 15$ juillet 1386.

ASL, Archivio notarii, $\mathrm{n}^{\mathrm{o}} 294, \mathrm{f}^{\mathrm{o}} 164 \mathrm{r}^{\circ}-165 \mathrm{v}^{\circ}, 8$ juin 1389.

${ }^{11}$ ASL, Archivio notarii, no 241, p. 94-96 (pagination moderne), 7 janvier 1367.

ASL, Archivio notarii, $\mathrm{n}^{\mathrm{o}}$ 123, vol. II, p. 5-6 (pagination moderne), 3 janvier 1375.

${ }^{12} \mathrm{Ce}$ qui pourrait expliquer l'absence quasi complète de comptes rendus des réunions où l'assemblée se contentait de délibérer, sans élire quiconque.

${ }^{13}$ Voir Paolo Prodi, Il Sacramento del potere. Il giuramento politico nella storia costituzionale dell'Occidente, Milano, Il Mulino, 1992.

${ }^{14}$ Sur la question du contractualisme politique, voir Avant le contrat social. Le contrat politique dans l'Occident médiéval, XIII ${ }^{e}-X V^{e}$ siècle, dir. François Foronda, Paris, Publications de la Sorbonne, 2011. Et plus précisément, Id., « Du contrat ou de la structure proprement politique des sociétés politiques», dans Avant le contrat social, op. cit., p. 5-13 et Patrick Boucheron, "L'Italie, terre de contrats », ibid., p. 17-23. 
particulier, l'exigence d'une justice équitable pour tous et la protection du bien commun, ici identifié aux propriétés collectives du quartier.

\section{Quartiers et paroisses}

Les différents actes étudiés montrent que des subdivisions de nature, de fonction et de taille différentes coexistaient à Lucques entre 1369 et 1400, parfois sous le même nom. Deux groupes principaux se dégagent : le premier est constitué des quartiers proprement dits, appelés bracchio ou contrada. La dernière rubrique des Statuts urbains de 1308 les définit comme des associations volontaires d'au moins dix habitants logeant dans des maisons voisines ${ }^{15}$. Ces circonscriptions avaient, au début du XIV $\mathrm{XI}^{\mathrm{e}}$ siècle, un rôle fiscal et politique. C'est en leur sein qu'étaient tirés au sort les électeurs des 550 membres du Conseil général et c'est également entre elles qu'étaient réparties les différentes charges fiscales. Elles étaient par ailleurs, comme le nom de contrada le suggère, des regroupements volontaires ${ }^{16}$, mais dont l'existence devait être reconnue par les autorités urbaines. Ces circonscriptions facilitaient également le contrôle de la population puisque c'est en leur sein que s'effectuaient les recensements dont témoignent les listes de population des années 1370-1372. À la fin du XIV ${ }^{\mathrm{e}}$ siècle toutefois, elles ont perdu leur rôle politique, puisque c'est désormais au niveau du Tiers que se fait la distribution des différentes charges publiques, et leur rôle fiscal est limité par le fait que la ville même de Lucques n'est pas soumise à une imposition directe ${ }^{17}$.

\footnotetext{
15 Statutum Lucani Communis. An. MCCCVIII [1867], éd. Salvatore Bongi et Leone Del Prete, Lucca, Maria Pacini Fazzi, 1991, Livre V, Rubrique LXVII, p. 329-330.

${ }^{16}$ Le terme de contrada, qui n'est pas propre à Lucques puisque c'est le même terme qui est par exemple employé à Venise, rappelle, lui aussi, par son étymologie la dimension contractuelle de ces circonscriptions territoriales.

${ }^{17}$ Sur la question des emprunts forcés auxquels la ville de Lucques doit soumettre sa population pour rembourser les emprunts contractés dans les années 1369-1370 pour
} 
Le deuxième groupe est quant à lui constitué des paroisses qui, souvent, recoupaient le territoire du quartier auquel elles donnaient leur nom, mais pas systématiquement comme le montre l'exemple de San Jacopo de Tumba ${ }^{18}$. Les cinq comptes rendus concernant les élections de ce quartier qui apparaissent dans les registres de Simone Alberti, Orso Barsellotti et Panfoglia Torringhelli permettent en effet de montrer la coexistence de ces deux formes d'association et leur interpénétration $^{19}$. Un des actes concerne la paroisse San Jacopo en tant que circonscription religieuse ${ }^{20}$, un le quartier à proprement parler $^{21}$ et trois une entité plus indéterminée, qui semble centrée sur la paroisse mais inclut également en partie le quartier. La première paraît, dans les années 1370, couvrir un territoire plus large que le second, ce dont témoigne le fait que les réunions de la paroisse comptent entre 27 et 34 personnes, quand celle de la contrada, le 18 janvier 1373, n'en rassemble que huit. Si nous postulons qu'un quorum des deux-tiers de présents était nécessaire, comme cela est précisé pour la paroisse, nous pouvons affirmer que le quartier comptait au maximum douze hommes en 1373 et la paroisse entre 40 et 51 entre 1379 et 1387. La comparaison de ces actes avec les listes de population des années 1370-1372 fait apparaître de manière encore plus claire l'enchevêtrement institutionnel qui caractérise

acquérir sa liberté, voir Christine Meek, Lucca, 1369-1400. Politics and society in an early Renaissance city-state, Oxford, Oxford University Press, 1978, p. 48-76.

${ }_{18}$ À Venise au contraire, comme l'a montré Élisabeth Crouzet-Pavan, les contrade, qui sont les circonscriptions administratives de base de la ville, ont fini par se confondre avec les paroisses. Voir Élisabeth Crouzet-Pavan, «Sopra le acque salse ». Espaces, pouvoir et société à Venise à la fin du Moyen Âge, Roma, École française de Rome, 1992, 2 vol., vol. 1, p. 554-561.

${ }^{19}$ Un contrat de location peut également être trouvé dans les registres de Panfoglia Torringhelli, signé entre les maîtres de la fabrique et procurateurs de l'église San Jacopo de Tumba, Francesco fils de feu Nuccorino et Piero Luporini, citoyens lucquois et tisserands de soie, et Nieruccio Bacciomei. Il s'agit d'un contrat de location de deux ans, avec un loyer de cinq florins d'or, daté du 25 mars 1385 (ASL, Archivio notarii, $\left.\mathrm{n}^{\mathrm{o}} 293, \mathrm{f}^{\mathrm{o}} 36 \mathrm{v}^{\circ}\right)$.

${ }^{20}$ ASL, Archivio notarii, $\mathrm{n}^{\mathrm{o}} 250, \mathrm{II}_{\mathrm{f}} \mathrm{f}^{\mathrm{o}} 84 \mathrm{v}^{\mathrm{o}}-85 \mathrm{r}^{\circ}, 15$ avril 1387.

${ }^{21}$ ASL, Archivio notarii, $\mathrm{n}^{\mathrm{o}} 262, \mathrm{f}^{\mathrm{o}} 39 \mathrm{v}^{\circ}-40 \mathrm{r}^{\circ}, 18$ janvier 1373. 
les environs de cette église. En 1371, deux listes, se référant à une contrada San Jacopo de Tumba, sont en effet déposées, qui semblent renvoyer à deux circonscriptions territoriales distinctes, quoique appartenant toutes deux au Tiers San Martino. La première a été déposée le 7 janvier 1371 par le consul Francesco Juntori, un habitant du quartier, et comprend six noms, dont quatre sont communs avec ceux contenus dans l'acte de $1373^{22}$. La deuxième a été déposée le 9 janvier de la même année, par le tisserand Bartolomeo Orsini, consul de la contrada qui ne fait pas partie de ses habitants, et compte elle trente noms ${ }^{23}$. Les deux listes ne partagent aucun patronyme en commun. Une comparaison entre ces deux listes et les noms contenus dans les différents actes notariés conservés confirme que ces deux quartiers devaient être situés dans le territoire de la paroisse San Jacopo de Tumba : des noms issus des deux quartiers sont récurrents dans les différentes réunions de cette dernière. Il semble possible que les habitants du plus petit des deux quartiers l'aient formé en faisant sécession du plus grand, comme le Statut de 1308 en offrait la possibilité ${ }^{24}$. Dans l'estime de 1399, une seule contrada San Jacopo de Tumba est enregistrée, avec les noms de quinze familles, parmi lesquels nous pouvons repérer des individus issus des deux quartiers de $1371^{25}$. Une hypothèse probable est donc que dans le dernier tiers $d u X^{\mathrm{e}}{ }^{\mathrm{e}}$ siècle, les deux quartiers se soient réunifiés, peut-être en raison de la diminution de leur population. Les trois actes des années 1380 conservés pourraient donc concerner ce quartier réunifié autour de sa paroisse et non simplement cette dernière.

\footnotetext{
${ }^{22}$ ASL, ms. 176, p. 47.

${ }^{23}$ Ibid., p. 69.

${ }^{24}$ Voir n. 4.

${ }^{25}$ Lucca, Biblioteca Statale, ms. 925, $\mathrm{f}^{\mathrm{o}} 249 \mathrm{r}^{\mathrm{o}}-258 \mathrm{r}^{\mathrm{o}}, \mathrm{f}^{\mathrm{o}} 257 \mathrm{r}^{\mathrm{o}}$. Le manuscrit date $\mathrm{du}$ $\mathrm{XVI}^{\mathrm{e}}$ siècle.
} 
La lecture des sources suggère qu'au-delà des considérations matérielles portant sur le territoire ou les propriétés collectives des différentes associations, ces dernières existent essentiellement par les hommes qui les composent, les représentent et, in fine, les incarnent. Les églises restent par ailleurs, à la fin du XIV siècle à Lucques, l'un des principaux pôles de la vie urbaine : autour d'elles s'organisent des entités variées, dont le territoire et la fonction se recoupent plus ou moins et auxquelles elles servent de point de repère et donnent le plus souvent leur nom. Qu'elles aient un caractère administratif ou au contraire religieux, ces réalités multiformes se définissent comme la concrétisation du rapport entre une communauté d'habitants et le territoire qu'elle occupe. En effet, comme l'écrit Pascal Vuillemin,

au XIII et au début du XIV ${ }^{\mathrm{e}}$ siècle, ce territoire et cette communauté constituaient, aux yeux des canonistes et plus généralement des contemporains, l'essence même de la paroisse. [...] L'existence de la paroisse et la construction progressive d'un droit paroissial dépendaient surtout de la conscience qu'avaient les paroissiens d'appartenir à un ensemble particulier, délimité, auquel ils se sentaient liés et à la vie duquel ils participaient ${ }^{26}$.

La définition des contrade et des brachii donnée par le Statut lucquois de 1308 plaçait également en son centre ce lien entre communauté d'habitants et territoire, puisqu'étaient nécessaires à la constitution d'une nouvelle entité administrative aussi bien l'union volontaire d'au moins dix voisins que la proximité de leurs habitations qui devaient soit être contiguës soit se faire face. Paradoxalement, c'est justement la composante territoriale de ces entités que nous connaissons le moins, les limites de la ville elles-mêmes faisant débat parmi les

\footnotetext{
${ }^{26}$ Pascal Vuillemin, «L'espace urbain vénitien. Un enjeu entre ordres mendiants et chapitres paroissiaux $\left(\mathrm{XIII}^{\mathrm{e}}-\mathrm{XV}^{\mathrm{e}}\right.$ siècle) », Rivista di storia della chiesa in Italia, $\mathrm{n}^{\mathrm{o}} 1$, 2009, p. 49-71, cit. p. 53.
} 
historiens ${ }^{27}$. Le territoire du quartier ou de la paroisse définissait l'espace sur lequel l'autorité de ces derniers s'exerçait. Il s'incarnait aussi bien dans la composante humaine de ces entités, qui nous apparaît dans les sources sous la forme des listes d'habitants ou des présents aux élections, que dans sa composante matérielle: les bâtiments et équipements qui étaient la propriété collective du quartier ou de la paroisse, et dont la gestion et la protection étaient l'une des tâches principales que les habitants déléguaient à leurs représentants. L'ensemble formé apparaît doté d'une personnalité juridique, qui le rendait susceptible d'être traduit devant n'importe laquelle des cours de justice de la ville, aussi bien civile qu'ecclésiastique.

Même si le caractère disparate de la documentation ne nous permet pas de nous faire une idée précise de la régularité et de la fréquence des réunions de ces associations, il parait clair que ces dernières n'avaient pas uniquement un rôle institutionnel abstrait dans la vie de leurs membres. Au moins une fois par an, les habitants se réunissaient pour procéder à l'élection de leurs représentants, en janvier pour les consuls, en juillet pour les procurateurs. Il ne s'agissait pas là de leur seule activité tangible. L'acte daté de 1387 évoque l'élection, par les habitants de San Jacopo de Tumba et les paroissiens de l'église du même nom, d'un remplaçant à leur prêtre. Il est clairement écrit qu'il s'agit là d'une prérogative desdits

\footnotetext{
${ }^{27} \mathrm{Si}$ le rattachement du plus grand nombre des quartiers à l'église du même nom permet d'en connaître grossièrement le centre et donc la localisation, leurs limites nous restent inconnues, car elles ne sont jamais précisées dans les sources. De ce fait, la dimension territoriale de la communauté et de la paroisse qui, sous la plume de Joseph Morsel, permet de les distinguer l'une de l'autre (la paroisse lui semblant être définie par rapport à son centre, l'église, et la communauté au contraire par ses limites), apparaît sensiblement identique dans la documentation, même s'il s'agit là d'un effet de sources. Voir Joseph Morsel, «Introduction », dans La Formation des communautés d'habitants au Moyen Âge. Perspectives historiograhiques, table ronde de Xanten, les 19-22 juin 2003, dir. Ludolf Kuchenbuch, Dieter Scheler, Joseph Morsel, en particulier p. 5-6, consultée en ligne le 22 juin 2015 : https://lamop.univparis1.fr/IMG/pdf/01_Introduction_Morsel_.pdf.
} 
habitants et paroissiens ${ }^{28}$, même si l'acte met également explicitement en valeur le contrôle du clergé lucquois sur la procédure ${ }^{29}$. Un autre acte évoqué plus haut, daté de 1373, évoque quant à lui une réunion de San Jacopo de Tumba convoquée par le consul Taluccio Nuchori pour rappeler l'obligation faite à ses habitants de participer à ces assemblées afin de discuter des affaires les plus importantes du quartier, notamment judiciaires. Même si aucun acte concernant une réunion de ce type n'a été trouvé, il apparaît clairement que ces associations ne reposaient pas uniquement sur l'action de leurs représentants, mais également sur la réunion régulière de l'assemblée de leurs membres, sur un modèle finalement assez proche de celui expérimenté à l'échelle de la ville.

Le gros de l'activité des quartiers reposait toutefois sur les épaules de leurs représentants. Ces derniers étaient de deux types : les consuls qui étaient élus par les contrade ou les brachii, recevaient un salaire et n'étaient pas forcément issus du quartier qu'ils représentaient; les procurateurs-syndics qui en étaient eux toujours issus, ne recevaient pas de salaire et représentaient en général la paroisse autant que le quartier proprement dit. Certains d'entre eux étaient d'ailleurs également maîtres de la fabrique de leur église. Ils étaient tous chargés de représenter l'entité qui les élisait devant les cours de justice de la ville et, plus généralement, de la défendre, comme ses membres, contre les dommages, principalement matériels, auxquels ils pouvaient être confrontés : étaient particulièrement visés les saisies, les amendes, les emprisonnements...

\footnotetext{
28 «Nominatio, electio seu praesentatio nomi rectoris tam de jure quam de consuetudine pertinet et spectat ad vicines et parrochianos dictae ecclesiae ». ASL, Archivio notarii, $\mathrm{n}^{\mathrm{o}} 250$, II, $\mathrm{f}^{\mathrm{o}} 84 \mathrm{v}^{\circ}$. «La nomination, l'élection ou le droit de présentation du nom du recteur appartient et incombe aux voisins et aux paroissiens de ladite église, aussi bien suivant le droit que suivant la coutume.» (nous traduisons).

${ }^{29}$ Il est en particulier précisé qu'après l'élection, le consul et les procurateurs du quartier vont la faire confirmer par l'évêque et le chapitre cathédral de la ville. Voir ibid., $\mathrm{f}^{\mathrm{o}} 85 \mathrm{r}^{\mathrm{o}}$.
} 
Les procurateurs avaient également dans leurs attributions la gestion des biens de l'association: entretien, mise en location et dans certains cas vente. Dans les listes de population des années 1370-1372, les consuls semblent eux jouer un double rôle qui les voit à la fois représenter et défendre le quartier d'un côté et servir de relais aux autorités urbaines de l'autre. Il y est en effet souvent précisé que ce recensement est adressé au podestat de la ville et à sa cour, ce qui laisse entendre que ce sont eux qui l'ont ordonné ${ }^{30}$. Si nous ignorons les motivations exactes qui ont poussé les autorités à réclamer ces listes ${ }^{31}$, il semble clair qu'elles avaient entre autres pour objectif de permettre le contrôle de la population lucquoise.

\section{Le lieu d'une première expérience politique à Lucques}

Le fonctionnement des associations de quartier repose donc en grande partie sur la participation plus ou moins active de leurs habitants, évidemment lors des réunions, mais également pour accomplir certaines tâches essentielles à la bonne organisation des quartiers. En effet, contrairement aux consuls, les syndics et procurateurs, les maîtres de la fabrique mais également les hommes choisis pour veiller au versement du salaire des consuls sont toujours des membres de la communauté

\footnotetext{
30 «Vannuccio Cincini consul dicte contrate dicto consulate nomine denumptiat et in scriptis dat vobis domino potestati et vostre curie omnes homines et personas habitantes in dicta contrata quorum nomina sunt hec videlicet ». ASL, ms.176, p. 23. «Vannuccio Cincini, consul de ladite contrade, du fait deladite fonction de consul, notifie et fournit par écrit, à vous seigneur Podestat et à votre cour, tous les hommes et toutes les personnes habitant dans cette contrade, dont les noms. sont comme il parait ci-dessous. » (nous traduisons).

31 Certaines formulations, comme celle vue dans la note précédente, semblent suggérer que ce recensement faisait partie des tâches annuelles du consul et n'avait donc rien d'exceptionnel, même s'il ne nous reste que les listes conservées dans les manuscrits 176 et 176 bis des archives, qui couvrent les années 1370 à 1372. Ces listes ne sont pas homogènes, ce qui ne permet pas d'être sûr de leur fonction exacte. Certaines signalent les habitants «inabiles », ce qui renvoie à la capacité à défendre la ville par les armes. D'autres mentionnent les veuves et les absents, ce qui pourrait laisser penser à une fonction fiscale et pas seulement politique.
} 
(habitants du quartier ou paroissiens). Comme nous l'avons vu plus haut, un acte concernant la contrada San Jacopo de Tumba nous rappelle qu'une telle participation n'allait pas forcément de soi et que, comme les principales institutions communales, au premier rang desquelles les grands Conseils urbains ${ }^{32}$, les différentes associations de quartier devaient sans doute souffrir d'un absentéisme chronique. Lors de la réunion du 18 janvier 1373 en effet, une amende de cinq sous lucquois est votée pour punir ceux qui ne se rendraient pas aux réunions et refuseraient de participer aux délibérations. Cet acte nous montre, a contrario, que la vie des quartiers est animée par leurs habitants et dépend en partie de leur bonne volonté. Un certain nombre de documents évoque également la solidarité financière qui s'impose à la population des quartiers, en particulier pour le paiement du salaire du ou des officiers élus mais aussi des amendes et autres obligations imposées par la commune ou l'un des tribunaux de la ville. Comme nous l'avons vu, les douze documents qui nous rapportent des élections de consuls mentionnent, même quand ce dernier est aussi un habitant du quartier, l'existence d'un salaire. Les habitants chargés de ce versement sont généralement qualifiés d'«imponitores» dans la documentation, et dans un cas $\mathrm{d}^{\prime}$ « $\operatorname{arbitrores}^{33} »$. Ils veillent à la bonne répartition de cette charge entre l'ensemble des voisins et sont responsables du paiement ${ }^{34}$. La fonction est

${ }^{32}$ Comme l'a montré Lorenzo Tanzini, la fréquence des réunions des conseils urbains
dans l'Italie de la fin du Moyen Âge, deux par semaine en moyenne à la fin du
XIII $^{\mathrm{e}}$ siècle, explique l'absentéisme important dont ces institutions souffrent. Quand
les sources permettent de l'évaluer, on estime qu'il devait tourner autour d'un tiers.
Voir Lorenzo Tanzini, A Consiglio. La vita politica nell'Italia dei comuni,
Roma/Bari, Laterza, 2014, p. $81-91$.
${ }^{33} \mathrm{ASL}$, Archivio notarii, $\mathrm{n}^{\mathrm{o}} 336, \mathrm{I}, \mathrm{f}^{\mathrm{o}} 71 \mathrm{v}^{\mathrm{o}}$, janvier 1394 .
${ }^{34}$ Ainsi, «Imponitores vero dicte contrate elligerunt, nominaverunt, fecerunt pro/per
salario dicti consulis imponendo hominibus et vicinis praedictis Johanem Puccinelli
et Guiglelmum Ser Marchesis suprascriptos praesentes et acceptantes dictam
impositionem»: ASL, Archivio notarii, $\mathrm{n}^{\mathrm{o}} 336$, III, $\mathrm{f}^{\mathrm{o}} 28 \mathrm{v}^{\mathrm{o}}-29 \mathrm{r}^{\mathrm{o}}$, 14 janvier 1394.
«Ils élirent, nommèrent, firent assignateurs de cette contrade, chargés d'assigner le 
donc d'importance et nécessite sans doute, outre une certaine capacité financière, de pouvoir exercer une forme d'autorité sur le reste de la collectivité. Peut-être dans le souci de garantir à la fois l'équité et l'efficacité de l'ensemble de l'opération, ces imponitores sont toujours au nombre de deux (ce que suggère également le terme «arbitrores »).

La comparaison des participants à la réunion convoquée par Taluccio Nucchori le 18 janvier 1373 avec les noms de la liste déposée deux ans auparavant par Francesco Juntori montre que la quasi-totalité des habitants devait être présente en 1373 : la liste de 1371 compte six noms ; celle de 1373, huit. Sur les six habitants mentionnés en 1371, cinq sont présents en 1373. Même si la rareté des sources empêche une évaluation précise, l'exemple souligne que la participation des habitants à la vie de leur quartier semble impliquer de larges franges de la population urbaine et constitue sans doute pour nombre d'entre eux une première, voire la seule, expérience politique lucquoise. Les documents consultés montrent en effet la variété des profils des citadins concernés par la gestion de leur quartier. Même si aucune femme n'apparaît dans la documentation, ces associations recrutent néanmoins plus largement que dans le simple cercle des citoyens. Plusieurs étrangers ${ }^{35}$ apparaissent en

paiement du salaire dudit consul aux hommes et voisins susnommés, Giovanni Puccinelli et Guglielmo Ser Marchesi susdits, ici présents et ayant accepté cette fonction. » (nous traduisons).

${ }^{35}$ La riche bibliographie sur les étrangers au Moyen Âge souligne l'hétérogénéité et donc l'imperfection de cette catégorie qui regroupe des individus fort divers. Sans trancher cette difficile question ici, nous nous contenterons dans ce paragraphe de désigner par le terme étranger des habitants de Lucques qui ne semblent pas y être nés, comme en témoignent aussi bien les mentions que les sources font parfois de leur origine géographique (comme les mots «du Portugal» ou «de Todi », même si de telles mentions peuvent être trompeuses à partir du moment où les patronymes deviennent héréditaires au cours du XIII ${ }^{\mathrm{e}}$ siècle) que les notations, plus explicites, sur le fait que tel individu, venu d'ailleurs, est désormais installé dans tel quartier de la ville. Voir "Arriver» en ville. Les migrants en milieu urbain au Moyen Âge, dir. Cédric Quertier, Roxane Chilà et Nicolas Pluchot, Paris, Publications de la Sorbonne, 2013 et particulièrement, Denis Menjot, «Introduction. Les gens venus 
effet dans ces actes et semblent jouir des mêmes droits et devoirs que les autres. Ainsi, le 24 juin 1384, à l'occasion de l'élection de nouveaux syndics dans la contrada San Jacopo de Tumba, l'acte notarié est enregistré devant deux témoins extérieurs à la communauté : Angelo fils de feu Giovanni de Todi, dont il est précisé qu'il demeure à Lucques, dans les faubourgs de la ville, et Lorenzo Nicolai, un fabriquant de lacets de Florence, habitant dans la contrada San Leonardo in Borgo ${ }^{36}$. Le 4 janvier 1394, lors de l'élection du nouveau consul de la contrada San Alessandro Minore, un certain Antonio Lorenzi de Crémone, dont il est précisé qu'il réside désormais à Lucques dans ladite contrada, est mentionné parmi les présents et donc les votants ${ }^{37}$. Enfin, un certain Antonio Barronci, de la commune de Florence, mais résidant désormais à Lucques, est élu consul d'au moins quatre quartiers différents au début de l'année 1394 : San Giovanni Maggiore le 11 janvier, San Domino le 13, San Giusto le 14, et San Martino, le 5 février.

Outre la place laissée aux étrangers, ces associations semblent se distinguer des principales institutions urbaines par leur hétérogénéité sociale forte, plus ou moins prononcée à l'échelle des différents quartiers, mais semble-t-il réelle à l'échelle de la ville tout entière, et en tout cas beaucoup plus marquée que dans les deux Conseils législatifs ou dans le Collège des Anciens lucquois. Cela se traduit dans certains quartiers par le fait qu'une partie voire la totalité des principales responsabilités est attribuée à des artisans. Ainsi, le 8 juin 1379, le tisserand Angelo Berti est élu syndic du quartier et de l'église San Jacopo de Tumba, en même temps que Michele Bernardi, dont le métier n'est pas précisé. En 1384, Jacopo Matucchi, tisserand, est cité comme maître de la fabrique de la

d'ailleurs dans les villes médiévales : quelques acquis de la recherche », p. 15-29 et Patrick Boucheron, «Conclusion. L'histoire en mouvement », p. 283-293.

${ }^{36}$ ASL, Archivio notarii, $\mathrm{n}^{\mathrm{o}} 292, \mathrm{f}^{\mathrm{o}} 117 \mathrm{r}^{\mathrm{o}}$.

${ }^{37}$ ASL, Archivio notarii, $\mathrm{n}^{\mathrm{o}} 336, \mathrm{III}, \mathrm{f}^{\mathrm{o}} 23 \mathrm{v}^{\mathrm{o}}-24 \mathrm{r}^{\mathrm{o}}, 4$ janvier 1394. 
même église, quand Giovanni Martini, Giovanni Luporini et Fiorensa Giovanni, tous les trois également tisserands, sont élus syndics et procurateurs par l'assemblée réunie le 24 juin. L'implantation de ces artisans dans leur quartier et dans la vie de leur église se constate dans la récurrence des fonctions qui leur sont confiées. Le 23 mars 1385, dans un contrat de location établi avec Nieruccio Bacciomei, ce sont deux autres tisserands de soie qui apparaissent en tant que maîtres de la fabrique et procurateurs de l'église San Jacopo de Tumba, Francesco Nucchorini et Piero Luporini, tandis que parmi les deux témoins de l'acte, dont il est précisé qu'ils sont tous les deux habitants du quartier, nous retrouvons le tisserand Angelo Berti, ainsi qu'un cultivateur, Orsuccio Orsucci ${ }^{38}$. Le 15 juillet 1386, Francesco Nucchorini et Piero Luporini sont toujours syndics et procurateurs de la contrada et de son église : ils ont donc dû être réélus au mois de juillet précédent. Ce jour-là, Francesco Nucchorini, Giovanni Luporini et Giovanni Martini sont réélus maîtres de la fabrique, en même temps que Nicolao Ser Pagani, marchand. Lors de la réunion du 15 avril 1387, qui vise à procéder à l'élection d'un nouveau prêtre pour l'église, Jacopo Matucchi apparaît comme consul de la contrada, et Giovanni Luporini et Giovanni Martini comme les deux maîtres de la fabrique. C'est sous leur direction qu'il est procédé à cette élection d'importance. Certains artisans participent donc de manière active et récurrente à la vie de leur quartier et à la gestion de ses principales institutions (en particulier son église), notamment dans les secteurs marqués par une forte présence artisanale, ce qui est le cas du quart nordest de la ville peuplé de nombreux tisserands, où San Jacopo de Tumba est situé. Cette participation s'appuie sur la maîtrise de compétences variées et étendues, en matière juridique et économique notamment. Sans

${ }^{38}$ Voir plus haut, n. 19. 
surprise, ceux qui accaparent les postes à responsabilité sont aussi ceux dont la présence aux réunions est la plus régulière.

La documentation permet également de déceler l'existence parmi les consuls de semi-professionnels. Il s'agit d'un groupe encore peu homogène, qui, à l'échelle locale, témoigne de l'utilisation de l'appareil d'État dans les processus d'ascension sociale à la fin du Moyen Âge ${ }^{39}$. Dans la majorité des cas, le consul n'est pas un habitant du quartier. Cette fonction est toujours rémunérée et fournit un revenu complémentaire, dans le cadre d'une pluriactivité populaire bien connue ${ }^{40}$. Ainsi, le 7 janvier 1367, le calandreur ${ }^{41}$ Paganuccio Bertini est élu consul de San Andrea in Pellaria, pour un salaire annuel de vingt-quatre livres ${ }^{42}$. Antonio Barronci est lui messager et crieur public de la commune à San Giovanni capo di Borgho. En tant que consul, il est payé dix livres par San Domino, seize par San Giusto et quatorze par San Martino.

La documentation permet donc également de prendre la mesure du nombre de ceux qui cumulent ces fonctions, qu'ils se fassent élire dans plusieurs quartiers en même temps comme Antonio Barronci, qu'ils soient régulièrement renouvelés dans leur poste comme Meo Gianni élu

\footnotetext{
${ }^{39}$ Sur cette question, voir Sandro Carocci, «Introduzione. La mobilità sociale e la “ congiuntura del 1300 ". Ipotesi, metodi di indagine, storiografia » dans La Mobilità sociale nel Medioevo, dir. Sandro Carocci, Roma, CEFR 436, 2010, p. 1-37.

40 Voir l'introduction, disponible sur internet, rédigée par François Menant pour présenter le séminaire «Les sociétés européennes au Moyen Âge: modèles d'interprétation, pratiques, langages » de l'année universitaire 2011-2012, en particulier les p. 28-30. François Menant, «Approches du peuple médiéval », introduction à l'année 2011-2012 du séminaire «Les sociétés européennes au Moyen Âge : modèles d'interprétation, pratiques, langages ", coorganisé à l'École Normale Supérieure avec Diane Chamboduc de Saint Pulgent: http://www.histoire.ens.fr/IMG/file/Menant/Introduction\%20séminaire\%2020112012,\%20Approches\%20du\%20peuple\%20au\%20Moyen\%20Âge.pdf.

Voir également Philippe Bernardi, Bâtir au Moyen Âge, XIII -milieu XVI siècle, Paris, CNRS, 2011, p. 20-24 et $I d$., "Le métier. Réflexions sur un mode d'identification », dans Le Technicien dans la cité en Europe occidentale. 1250-1650, dir. Mathieu Arnoux et Pierre Monnet, Roma, CEFR 325, 2004, p. 93-107, p. 94-95.

${ }^{41}$ Un calandreur avait pour tâche de lustrer les draps entre deux cylindres.

${ }^{42}$ ASL, Archivio notarii, $\mathrm{n}^{\mathrm{o}}$ 241, p. 94-95 (pagination moderne).
} 
consul de Santa Maria Filii Corbi en 1370 et $1371^{43}$, ou les deux à la fois. Les listes de population des années 1370-1372 permettent ainsi de recenser 71 consuls différents pour 83 quartiers. Bartolomeo del Maestro Giovannino est l'un des plus actifs. Il est consul de San Andrea in Pellaria en 1366 puis en $1375^{44}$. Il présente par ailleurs quatorze listes de population pour onze quartiers différents : dans le Tiers San Martino, il est ainsi consul de San Anastasio en 1370 et 1371, San Andrea en 1370 et 1371, San Simone e Juda en 1370 et 1371, Santa Giulia en 1370 et 1371, ainsi que des brachii Altiberi en 1370 et 1371, Figlioli Giordani, Passi, San Lorenzo Corvareja et San Michele de Guinizinghi et San Michele Venesinghi en $1371^{45}$. Dans le Tiers de San Salvatore, il est consul de San Pietro Cigholi en 1370 et de San Andrea in Pellaria en 1370 et $1371^{46}$. Les quartiers semblent souvent reconduire les consuls d'une année sur l'autre. Danino Gucci est ainsi réélu consul de trois quartiers en 1370 et $1371^{47}$. Au total, vingt-quatre quartiers sur quatre-vingt-trois élisent au moins deux fois le même consul entre 1370 et 1372 .

À la fin du XIV ${ }^{\mathrm{e}}$ siècle, les associations de quartier apparaissent donc comme le premier échelon des institutions politiques lucquoises. Chargées de gérer la vie quotidienne et les possessions communes desdits quartiers, elles sont un lieu d'intégration de larges franges de la population à la communauté politique urbaine, au-delà même du cercle des citoyens, puisqu'y participent nombre d'étrangers. Leur étude permet donc de nuancer en partie le constat de Christine Meek d'une fermeture

\footnotetext{
${ }^{43}$ Ibidem, p. 130 et ms. 176 bis, $\mathrm{f}^{\mathrm{o}} 15 \mathrm{v}^{\mathrm{o}}-16 \mathrm{r}^{\mathrm{o}}$.

${ }^{44}$ Voir n. 11.

45 ASL, ms. 176 bis, f $\mathrm{f}^{\mathrm{o}} 3 \mathrm{r}^{\mathrm{o}}$; ms. 176, p. 52 ; ms. 176 bis, $\mathrm{f}^{\mathrm{o}} 2 \mathrm{r}^{\mathrm{o}}$; ms. 176, p. 198 ; ms. 176 bis, $\mathrm{f}^{\mathrm{o}} 2 \mathrm{r}^{\mathrm{o}}$; ms. 176 , p. 51 ; ms. 176 bis, $\mathrm{f}^{\mathrm{o}} 1 \mathrm{v}^{\mathrm{o}}$; ms. 176, p. 50 ; ms. 176 bis, $\mathrm{f}^{\mathrm{o}} 1 \mathrm{r}^{\mathrm{o}}$; ms. 176, p. 197 ; ibid., p. 49.

${ }^{46}$ ASL, ms. 176 bis, $\mathrm{f}^{\circ} 4 \mathrm{r}^{\circ}$ et $4 \mathrm{v}^{\circ}$.

47 ASL, ms. 176, p. 77 ; ms. 176 bis, $\mathrm{f}^{\mathrm{o}} 49 \mathrm{v}^{\mathrm{o}}$; ms. 176, p. 93 ; ms. 176 bis, f $\mathrm{f}^{\mathrm{o}} 50 \mathrm{v}^{\mathrm{o}}$; ms. 176 , p. 91 ; ms. 176 bis, $\mathrm{f}^{\mathrm{o}} 48 \mathrm{v}^{\mathrm{o}}$.
} 
de la vie politique urbaine ${ }^{48}:$ si cette oligarchisation semble indéniable à l'échelle des institutions centrales, elle n'empêche pas l'implication réelle d'une bonne partie de la population dans la vie politique quotidienne de la ville, dont le quartier est l'un des cadres. De surcroît, ces associations sont le témoin de la diffusion de la culture politique communale dans la population lucquoise, qui y manifeste son attachement aux valeurs du Popolo, comme la défense du bien commun et d'une justice équitable pour tous. Enfin, les quartiers sont l'un des lieux d'expression du politique à Lucques, notamment au sein de la milice urbaine : ainsi, c'est d'une des compagnies de cette dernière que part le mouvement de destruction de l'Augusta en mars 1370, symbole de la tyrannie mise à bas dès la liberté récupérée ${ }^{49}$. Cette initiative souligne aussi bien la diffusion de cet idéal de liberté dans la population urbaine que le rôle joué par les quartiers dans l'expression du politique. Cette intégration de larges franges de la population à la politique urbaine n'est toutefois possible que parce que ces processus excluent autant qu'ils incluent: à la fin du $\mathrm{XIV}^{\mathrm{e}}$ siècle, les femmes restent ainsi absentes des associations de quartier comme des institutions centrales de la République lucquoise.

\footnotetext{
${ }^{48}$ Christine Meek, Lucca, 1369-1400, op. cit., p. 170-193.

${ }^{49}$ Giovanni Sercambi, Croniche, éd. Salvatore Bongi, Roma, Istituto Storico Italiano, 1892, 3 vol., vol. I, chap. CCXIV, p. 187.
} 